\title{
Lazer entre universitários da área da saúde: revisão de literatura
}

\author{
Leisure among university students in the healthcare area: \\ a review of the literature
}

Júlia Lelis Vieira ${ }^{1}$

Liana Abrão Romera ${ }^{2}$

Maria Cristina Pereira Lima ${ }^{1}$

${ }^{1}$ Faculdade de Medicina, Universidade Estadual Paulista Julio de Mesquita Filho. Rubião Junior s/n, Rubião Junior. 18618000 Botucatu SP Brasil. profjuliavieira@gmail.com ${ }^{2}$ Centro de Educação Física e Desportos, Centro de Ciências da Saúde, Universidade Federal do Espírito Santo. Vitória ES Brasil.
Abstract This article set out to analyze studies on leisure activities among university students in the healthcare area. It conducted a search using the following key words: leisure and students, and their corresponding terms in Spanish and English in the Bireme database, which resulted in 32 articles at the end of the review. In the articles analyzed there was a predominance of the following leisure interests: sports, social, intellectual and artistic activities, with a greater number of articles presenting experiences in sports. It was also noted that the intake of alcohol in the leisure context is often mentioned by this population group. There was a predilection for physical exercised/ sport during leisure time, compared to other leisure interests and little knowledge about any other possibilities for leisure. This article also discusses the need to develop strategies that promote leisure among these students, seeking to improve their quality of life. It is noteworthy that they are health professionals in the training phase and it is quite possible that their future orientations might be influenced by their personal practices.

Key words Leisure activities, Students, Mental health, Alcohol abuse
Resumo Esse artigo objetiva analisar estudos sobre lazer entre universitários da área da saúde, a partir de revisão da literatura. Utilizou-se os descritores: lazer e estudantes, e seus respectivos termos em espanhol e inglês na base de dados Bireme, resultando no final da revisão em 32 artigos. Observou-se um predomínio dos seguintes interesses do lazer: desportivos, sociais, intelectuais e artísticos, com maior número de artigos apresentando vivências nos esportes. Notou-se também que a ingestão de álcool no âmbito do lazer é bastante mencionada por esta população. Há uma predileção por práticas corporais no tempo livre, em relação aos demais interesses no contexto do lazer, havendo pouco conhecimento sobre os diferentes conteúdos do lazer. Discute-se a necessidade de criar estratégias que incentivem o lazer entre os estudantes para melhorar a qualidade de vida. Ressalta-se que são profissionais da saúde em formação e é possível que suas orientações futuras sofram influência de suas práticas pessoais.

Palavras-chave Atividades de lazer, Estudantes, Saúde mental, Abuso de álcool 


\section{Introdução}

Existe um consenso de que a prática do lazer seja um importante meio de diminuição das tensões geradas pela sobrecarga no dia a dia, além de um momento de descanso. Embora associado a tais ideias, o lazer pode significar muito mais do que isto. A palavra lazer é originada do latim - licere -, e tem por significado "ser lícito", "ser permitido", "poder" "ter o direito".

Segundo Norbert e Dunning2: "[...] todas as atividades de lazer são atividades de tempo livre, mas nem todas as de tempo livre são de lazer... as características especiais das atividades de lazer só podem ser compreendidas se forem consideradas, não apenas em relação ao trabalho profissional, mas também, em relação às várias atividades de não lazer, no quadro de tempo livre". Os autores diferenciam o trabalho profissional e o trabalho não profissional, considerando o primeiro como associado à remuneração, enquanto que o segundo relaciona-se a todas as outras atividades da vida. Deste modo, o trabalho não profissional, embora realizado no tempo livre, não compreenderia lazer, necessariamente.

Martins e Batista ${ }^{3}$ criticam a evolução social, cultural e financeira que teria transformado o lazer nos dias atuais em um produto, perdendo sua essência. Nas palavras dos autores: "o mundo hipermoderno tomado pelo consumismo termina por influenciá-lo (lazer) de forma a deteriorá-lo, mercantilizá-lo, coisificando-o e empobrecendo -o de significados"3.

Dentro da lógica capitalista, o lazer tende a ser visto como uma vivência supérflua, desnecessária ${ }^{3}$. No entanto, no que diz respeito à saúde, tais vivências têm sido vistas como importantíssimas, pois permitem que ocorram a construção de relações de vínculos, a corresponsabilidade e a autonomia com a própria saúde ${ }^{4}$.

Alguns autores têm tentado classificar as atividades compreendidas como lazer, segundo os, assim chamado, conteúdos do lazer: físico-esportivo, artístico, manual, social e intelectual, propostos por Dumazedier ${ }^{5}$ e turístico, proposto por Camargo ${ }^{6}$. Discute-se a inclusão nesta lista o conteúdo "virtual", referindo-se ao uso da internet e jogos eletrônicos ${ }^{7}$. De um modo geral, na literatura, são encontradas diversas publicações que associam melhor saúde física e/ou mental à vivência do lazer na população em geral ${ }^{8-10}$, destacando-se a população jovem e os estudantes de graduação $^{11-14}$.

Considerando pesquisas que têm apontado o período de graduação como um momento tenso na vida dos estudantes e marcado por diferentes formas de sofrimento psíquico ${ }^{15-17}$, este estudo tem como objetivo apresentar uma revisão da literatura das pesquisas que avaliaram lazer entre estudantes universitários da área da saúde. Este tema se mostra relevante na medida em que o lazer poderia consistir em uma estratégia de enfrentamento ao sofrimento psíquico experimentado pelos estudantes e um recurso a ser estimulado entre seus futuros pacientes.

\section{Metodologia}

A revisão de literatura deu-se por uma pesquisa no banco de dados Bireme, que integra as bases Medline e Lilacs, realizada em julho de 2015. A pesquisa utilizou as terminologias cadastradas nos descritores em Ciências da Saúde da Biblioteca Virtual em Saúde, tendo sido utilizados os seguintes descritores: lazer e estudantes, e seus respectivos termos em espanhol e inglês. Outros descritores foram associados a estes, na forma de filtros, para refinar a busca, sendo eles: atividades de lazer, estudantes de medicina, estudantes de enfermagem, qualidade de vida, universidades, estilo de vida, educação médica, currículo, educação em enfermagem, recreação, satisfação pessoal, leitura, estudantes de farmácia, instituições acadêmicas, escolha da profissão, carga de trabalho e saúde mental. Foram utilizadas as ferramentas disponíveis AND/OR da base de dados.

Para a inclusão, foram utilizados os seguintes critérios: (A) pesquisas que se referissem a estudantes de graduação na área da saúde; (B) investigações que pesquisassem lazer em qualquer modalidade incluída entre os conteúdos do lazer; (C) artigos publicados nas línguas: espanhol, inglês e português.

A pesquisa se deu em três etapas: (1) leitura dos títulos dos artigos e seleção dos resumos; (2) leitura dos resumos e seleção daqueles cujos artigos completos seriam buscados; (3) leitura na íntegra dos artigos que se encaixavam no critério de inclusão da pesquisa (Figura 1).

\section{Resultados e Discussão}

Inicialmente, como explicado na metodologia, os descritores foram inseridos na base de dados obtendo um resultado de 690 títulos relacionados à pesquisa. Seguindo os passos, cada título foi lido selecionando-se 91 para a leitura dos resumos. Três títulos foram excluídos pois não apresenta- 


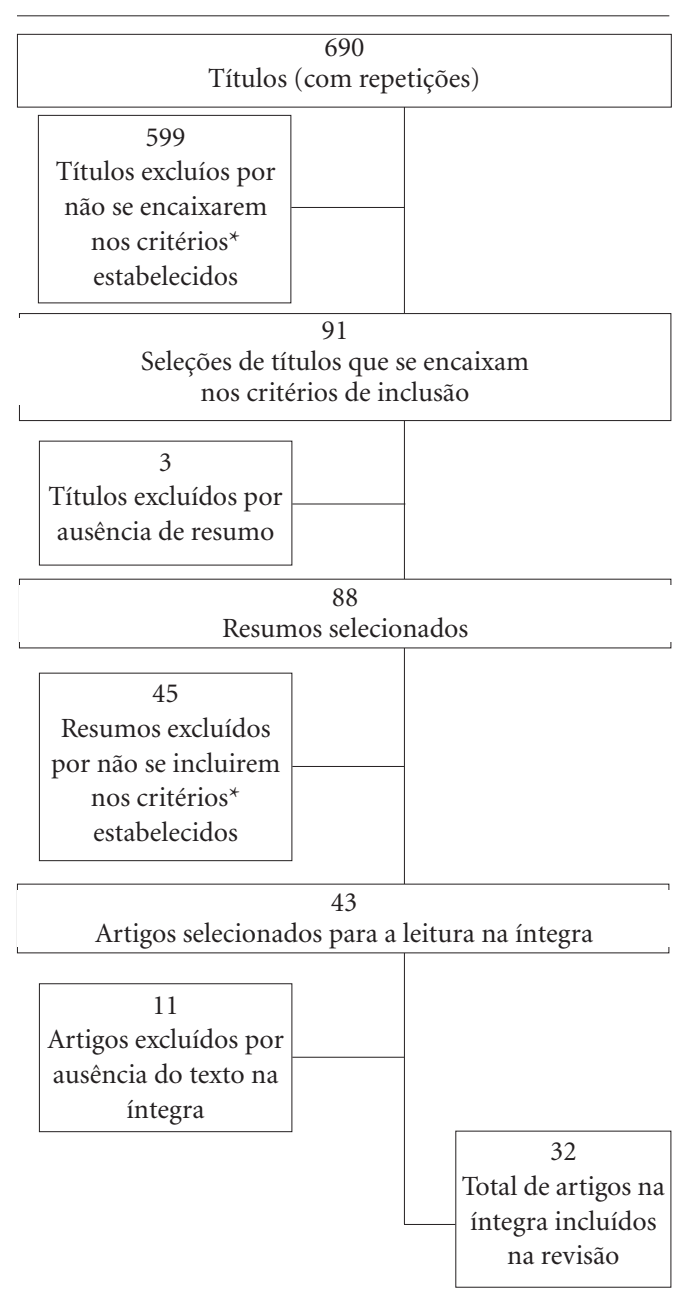

Figura 1. Organograma da seleção dos artigos incluídos na revisão.

*(A) pesquisas que se referissem a estudantes de graduação na área da saúde; (B) investigações que pesquisassem lazer em qualquer modalidade incluída entre os conteúdos do lazer; (C) artigos publicados nas línguas: espanhol, inglês e português.

vam resumos. Posteriormente à leitura dos resumos, 43 artigos foram escolhidos para a leitura na íntegra; entretanto 11 foram eliminados por não ser encontrado o texto completo, finalizando a busca com 32 artigos completos.

Os artigos foram lidos observando-se os seguintes aspectos: tipo de estudo, vivências de lazer investigadas; curso e país no qual o estudo foi desenvolvido, destacando-se os principais achados. Dentre os 32 artigos, quinze se referiam a estudos realizados no Brasil e desessete em outros países: Estados Unidos, México, Espanha, Colômbia, Cuba, Inglaterra e Reino Unido. Para facilitar a visualização, os artigos foram distribuídos em três quadros: aqueles produzidos no Brasil (Quadro 1), aqueles produzidos em outros países (Quadro 2) e aqueles que, independentemente de onde tenha sido produzidos, discutiam o papel do álcool para os estudantes no lazer (Quadro 3).

Embora o lazer seja constituído por sete conteúdos $^{5-7}$, chama a atenção que na literatura predominem investigações sobre a prática corporal no contexto do lazer. É possível que esse predomínio esteja relacionado à maior facilidade de mensuração da atividade, por exemplo, em termos do número de horas e vezes por semana que a atividade física pode ser descrita. Outra possibilidade é que, como a prática esportiva tem sido associada a benefícios atribuídos aos efeitos fisiológicos sobre o corpo humano, acabe sendo mais estudada ${ }^{18-23}$.

Estudos mostram que praticar exercício físico regularmente e de variadas intensidades tem-se associado a melhor condicionamento físico, liberação de beta-endorfinas - sensação de bem-estar, diminuição de glicemia e pressão arterial e aumento de massa óssea e magra ${ }^{24-27}$.

Entre as atividades físicas mais comumente investigadas está a musculação, atividade corporal que apresenta benefícios tanto no que diz respeito a ganho de massa magra e diminuição de taxas glicêmicas quanto à melhora do condicionamento cardiorrespiratório ${ }^{24-26}$. Essa prática corporal está associada a variáveis como: renda, gênero, idade, período do curso ${ }^{18,20,28}$. Além dos benefícios fisiológicos já mencionados, observou-se nos achados a valorização do componente social, corroborando estudos desenvolvidos em uma universidade do interior de São Paulo ${ }^{29,30}$.

Outras modalidades também têm sido investigadas no âmbito do esporte como futebol, como caminhada e/ou corrida. O futebol tem grande influência da cultura nacional, enquanto caminhada e/ou corrida são práticas corporais que não necessitam de grandes investimentos financeiros, e com benefícios para saúde física e mental. Entretanto, o gênero ainda tem sido uma variável significativa, visto que homens tendem a praticar mais tais modalidades que mulhe$\operatorname{res}^{18,19,23}$.

Há estudos mostrando que ao longo do tempo, no curso de medicina, existe uma tendência à diminuição das atividades físicas e também de atividades de lazer e recreação ${ }^{21}$. Sousa et al. ${ }^{28}$ também observaram no Brasil que, durante o curso, os estudantes universitários aumentam a prevalência de condutas negativas à saúde: dimi- 
Quadro 1. Características dos estudos com estudantes universitários na área da saúde no Brasil associados a atividades no âmbito do lazer.

\begin{tabular}{|c|c|c|c|c|c|}
\hline Autor & Ano & $\begin{array}{l}\begin{array}{l}\text { Tipo de } \\
\text { estudo }\end{array} \\
\end{array}$ & Amostra & $\begin{array}{c}\text { Público } \\
\text { Curso }\end{array}$ & Cidade/Estado \\
\hline Vieira, E.M., et al. & 2004 & Transversal & 686 & Medicina & São Paulo/SP \\
\hline Beuter, M., et al. & 2005 & $\begin{array}{l}\text { Descritiva } \\
\text { exploratória }\end{array}$ & 53 & Enfermagem & Santa Maria/RS \\
\hline Zonta, R., et al. & 2006 & Qualitativo & 25 & Medicina & Santa Catarina/SC \\
\hline Bielemann, R., et al. & 2007 & Transversal & 221 & Educação Física & Pelotas/RS \\
\hline Rocha, G.W.F.; Siqueira, H. & 2009 & Empírica & & Medicina & Rio de Janeiro/RJ \\
\hline Amaducci, C.M., et al. & 2010 & Transversal & 189 & Enfermagem & São Paulo/SP \\
\hline Fiorotti, K.P., et al. & 2010 & Transversal & 229 & Medicina & Espírito Santo/ES \\
\hline Meilke, G.I. et al. & 2010 & Transversal & 485 & Estudantes - diversas áreas & Pelotas/RS \\
\hline Baldissera, V.D.A.; et al. & 2011 & Qualitativa & 8 & Enfermagem & Maringá/PR \\
\hline Guareschi, N.M.F., et al. & 2011 & Descritivo & 23.613 & Psicologia & Brasil \\
\hline Sousa, T.F. & 2012 & Transversal & 1.084 & Estudantes ${ }^{1}$ & Bahia/BA \\
\hline Tempski et al. & 2012 & Qualitativo & 56 & Medicina & Brasil $^{2}$ \\
\hline Sousa, T.F., et al. & 2013 & Transversal & 5.461 & Estudantes $^{1}$ & Bahia/BA \\
\hline
\end{tabular}

Quadro 2. Características dos estudos com estudantes universitários na área da saúde no âmbito internacional associados a atividades no âmbito do lazer.

\begin{tabular}{|c|c|c|c|c|c|}
\hline Autor & Ano & $\begin{array}{l}\text { Tipo de } \\
\text { estudo }\end{array}$ & Amostra & $\begin{array}{l}\text { Público } \\
\text { Curso }\end{array}$ & País \\
\hline Kelly, J.A., et al. & 1982 & Longitudinal & 48 & Medicina & EUA \\
\hline Wolf, T.M.; Kissling, G.E. & 1984 & Longitudinal & 104 & Medicina - calouros & EUA \\
\hline Folse, M.L., et al. & 1985 & Transversal & 71 & Medicina & EUA \\
\hline Rosenthal, T.L. et al. & 1989 & $\begin{array}{l}\text { Validação de } \\
\text { instrumento }\end{array}$ & 670 & $\begin{array}{l}\text { Estudantes universitários de } \\
\text { Psicologia introdutória }\end{array}$ & EUA \\
\hline Bradby, M.; Soothill, K. & 1997 & Longitudinal & 444 & Enfermagem & Inglaterra \\
\hline $\begin{array}{l}\text { Hodgson, K.; Thomson, } \\
\text { R. }\end{array}$ & 2000 & Transversal & $\begin{array}{l}384 \\
151 \\
137\end{array}$ & $\begin{array}{l}\text { Medicina } \\
\text { Biologia } \\
\text { Direto }\end{array}$ & Inglaterra \\
\hline Haase A. et al. & 2004 & Transversal & 19.298 & Estudantes & 23 países \\
\hline Marshall, L.L., et al. & 2008 & Transversal & 109 & Farmácia & EUA \\
\hline Sanches, J.R.L. & 2008 & Observacional & 150 & Psicologia & Cuba \\
\hline Soto, L., et al. & 2009 & Transversal & 598 & Estudantes & Colômbia \\
\hline Salazar-Torres et al. & 2010 & $\begin{array}{l}\text { Validação } \\
\text { instrumento }\end{array}$ & $\begin{array}{l}1^{\circ} 1485 \\
2^{\circ} 1811\end{array}$ & Estudantes & Colômbia \\
\hline $\begin{array}{l}\text { Lonsdale, A.J.; North, } \\
\text { A.C }{ }^{1} \text {. }\end{array}$ & 2011 & Transversal & $\begin{array}{l}300 \\
117 \\
189 \\
700\end{array}$ & $\begin{array}{l}\text { Universitários } \\
\text { Psicologia } \\
\text { Psicologia } \\
\text { Voluntários de várias idades }\end{array}$ & Reino Unido \\
\hline Molina-Garcia, J. et al. & 2011 & Transversal & 639 & Estudantes & Espanha \\
\hline Carballeira, M., et al. & 2015 & Transversal & 346 & Psicologia & México e Espanha \\
\hline
\end{tabular}

${ }^{1}$ Publicação contendo quatro estudos, sendo quatro grupos amostrais.

nuem as atividades físicas no lazer e aumentam o consumo de bebidas alcoólicas, entre outras.
Chamou a atenção nos achados desta revisão alguns estudos cujo conteúdo no âmbito do la- 
Quadro 3. Características dos estudos do uso de álcool no tempo livre entre os universitários na área da saúde.

\begin{tabular}{lclrll}
\hline \multicolumn{1}{c}{ Autor } & Ano & \multicolumn{1}{c}{ Tipo de estudo } & Amostra & Público & \multicolumn{1}{c}{ País } \\
\hline Mesquita, A.M.C. et al. & 1995 & Quanti-qualitativo & 1.080 Universitários & Brasil \\
Correia, C.J. et al. & 2003 & Tranversal & 256 Universitários & EUA \\
Fournear, A.K. et al. & 2004 Quase experimental & 356 Universitários & EUA \\
Glindemman, K.E., et al. & 2007 & Experimental & 702 Universitários & EUA \\
Finlay, A.K., et al. & 2012 Longitudinal & 717 Universitários & EUA \\
\hline
\end{tabular}

zer foi o intelectual, artístico e social. Atividades como, leitura, estudar e /ou ouvir música, teatro, assistir TV, frequentar ambientes sociais como os espaços do centro acadêmico e/ou esportivos, foram todos mencionados como atividades desenvolvidas no tempo livre ${ }^{31-36}$. Tais modalidades podem estar associadas aos benefícios fisiológicos que regulam o humor e o estado de bem-estar, além do apoio social ${ }^{27,29}$.

Estudos mostraram que tais vivências, além do benefício próprio, também têm uma conotação acadêmica, pois práticas como o aprendizado de uma língua estrangeira, música e teatro melhorariam o currículo do estudante no futuro, podendo interferir, por exemplo, na seleção para a residência médica ${ }^{31,33}$.

Com o intuito de pesquisar as percepções acerca do lazer, Baldissera et al. ${ }^{37}$ observaram diferenças sobre o lazer na vida pessoal do estudante de enfermagem e na vida profissional além da compreensão do senso comum atribuído ao lazer. Na Inglaterra, Bradby e Soothill ${ }^{38}$ observaram que estudantes de enfermagem apresentavam uma grande variedade de experiências no contexto do lazer. Beuter et al. ${ }^{32}$ estudaram o contexto do cuidado de si e do outro entre estudantes da mesma categoria e observaram que as atividades de lazer mais vivenciadas pelos estudantes estavam dentro do conteúdo artístico, intelectual e social, sendo: ouvir música e assistir tv. Para eles, os estudantes deveriam vivenciar mais amplamente os demais conteúdos do lazer com o intuito de trabalhar o corpo, a mente, a destreza manual, além das relações sociais, e, a partir da experiência pessoal, transportar tais vivências para o campo de atuação profissional.

Qualidade de vida e estresse e sua relação com as atividades de lazer também foram investigados, em amostras de estudantes universitários. Os estudos encontrados observaram uma tendência a mostrar uma diminuição de estresse e melhor qualidade de vida nos sujeitos que viven- ciam algum tipo de atividade no âmbito do lazer e outros estudos apenas citaram o lazer como estratégias de enfrentamento ao estresse $\mathrm{e}^{11,21,39-43}$. Em um estudo qualitativo os alunos do curso de Medicina da Universidade Federal de Santa Catarina apresentaram como estratégias de enfrentamento ao estresse a valorização dos relacionamentos interpessoais, equilíbrio entre estudo e lazer, prática de atividade física, entre outras como organização do tempo, cuidados com a saúde, alimentação e o sono, religiosidade, e trabalhar a própria personalidade diante de situações adversas assim como procurar ajuda psicológica ${ }^{11}$. Amaducci et al. ${ }^{39}$ observaram que a estratégia mais abordada dos alunos para diminuição da fadiga foram sono de qualidade $(64,5 \%)$ e lazer $(63,5 \%)$, além de outras como descansar ou relaxar, práticas esportivas, tomar banho, práticas religiosas, entre outras menos citadas pela amostra estudada.

Kelly et al. ${ }^{41}$ acreditavam que o estudante precisava aprender técnicas de relaxamento, aprendizado de organização das prioridades, técnicas de modificação cognitiva, assim como planejamento do tempo para o lazer com o intuito de minimizar o estresse causado pela sobrecarga do curso. Outros estudos também discutem a necessidade de ensinar estratégias de enfrentamento ao estresse causado pela extensa grade curricular dos cursos de ensino superior ${ }^{40,42}$. Marshall et al. ${ }^{43}$ observaram que atividades como correr, ter tempo para a família e amigos, cochilar e/ou dormir, assistir TV e consumir álcool foram estratégias apontadas pelos estudantes de farmácia nos EUA para o enfrentamento do estresse e uma melhor qualidade de vida. É importante destacar que os autores não avaliaram se a associação com o uso de álcool não estaria, na verdade relacionada com rede de relacionamentos.

Assim como a associação de qualidade de vida e lazer entre universitários, foi encontrada na literatura a associação entre estilo de vida e saúde. Pesquisa realizada na Colômbia, cujo ob- 
jetivo foi investigar o estilo de vida em dez dimensões, observou associação entre práticas saudáveis e satisfação com estilo de vida e saúde em geral, sendo que a única exceção foi exercício e atividade física ${ }^{44}$. Fiorotti et al. ${ }^{17}$, ao descreverem o perfil do estudante de medicina, mostraram que este é um público de nível socioeconômico e cultural elevado, o que em geral está associado a maior disponibilidade de atividades de lazer. Contudo, o que os autores encontraram foram estudantes sobrecarregados devido à extensa grade curricular e que relatavam ter pouco tempo para o lazer. Corroborando os achados de Fiorotti et al. ${ }^{17}$, Sanches ${ }^{45}$ detectou entre os estudantes de psicologia em uma universidade de Cuba a necessidade e a posterior implantação de um programa de recreação organizado com estrutura física, profissionais habilitados e materiais disponíveis.

Haase et al. ${ }^{22}$ pesquisaram em 23 países a questão da inatividade física no lazer e observaram que havia níveis de prática corporal abaixo do recomendado, apesar de uma forte crença quanto ao comportamento saudável entre as culturas.

Um estudo realizado com estudantes de psicologia do México e da Espanha objetivou investigar as diferenças de bem-estar dentro das duas culturas - coletivista e individualista, resultando a valorização de ambas para a satisfação sentimental e vital. Segundo os autores, observou-se que a cultura mexicana valoriza o bem-estar subjetivo global e a satisfação com a saúde enquanto a cultura espanhola valoriza a satisfação com o ócio ${ }^{46}$.

Algumas pesquisas tiveram por objetivo a criação e validação de um instrumento específico para a mensuração das vivências no contexto cultural do lazer ${ }^{47,48}$.

Em 1989, Rosenthal et al. ${ }^{47}$ observaram que o instrumento denominado de leisure interests checklist - LIC apresentou grande potencial para utilização na descrição de interesses do lazer. Em 2010 na Colômbia foi validado um instrumento para avaliar o estilo de vida de jovens universitários em oito dimensões, sendo elas: atividade física, tempo de lazer, alimentação, consumo de álcool, cigarros e drogas ilegais, sono, habilidade interpessoal e enfrentamento no estado emocio$n a l^{48}$. Esse instrumento apresentou propriedades psicométricas adequadas, sendo avaliado como adequado para identificação das necessidades dos jovens universitários na Colômbia, assim como um instrumento norteador para ações voltadas a promoção e prevenção dentro das universidades $^{48}$.
Observando a associação do consumo de álcool no lazer dos universitários, foram encontrados artigos que discutem o uso de bebidas alcoólicas no tempo livre dos universitários e os fatores a este associado (quadro 3). Entre os estudantes de medicina da Universidade de São Paulo, na década de 90 , Mesquita et al. ${ }^{49}$ observaram que frequentar bares como atividades de lazer, entre outros aspectos, são fatores de risco para o aumento do consumo de substâncias psicoativas. Finlay et al..$^{50}$ observaram que havia maior propensão ao uso de álcool por estudantes que frequentavam as atléticas e que passavam mais tempo em atividades sociais enquanto havia menor uso de álcool por estudantes que frequentam encontros religiosos ou voluntários. Fournear et al..$^{51}$ e Glindemman et al. ${ }^{52}$ observaram que o uso de intervenção incentivo/recompensa nas festas em fraternidades era uma ferramenta muito positiva em favor da diminuição do consumo de álcool nas atividades sociais no contexto do lazer.

A associação entre consumo de álcool no tempo livre na vida dos estudantes encontrados nesta revisão bibliográfica ${ }^{28,50-52}$ confirmam os achados encontrados em pesquisas anteriores $^{53-61}$. Esses dados intensificam a necessidade da criação de estratégias para o enfrentamento ao uso nocivo de tal substância objetivando melhor saúde para os estudantes.

Entre os estudos revisados há aqueles que apontam os resultados negativos para a saúde, associados de algum modo à falta de lazer entre os estudantes universitários s $^{11,17,39,40,42,43,45}$.

Por conta de todas as evidências que apontam para pior saúde física e/ou mental associado à falta de lazer, é importante encontrar estratégias para minimizar tais riscos. Uma possibilidade talvez seja difundir entre os estudantes o modelo de habilidades do ócio ${ }^{62}$, fortalecendo a proposta de Beuter et al. ${ }^{32}$ de inserir na formação dos universitários o conceito do lazer e a teoria da recreação terapêutica. Esta estratégia objetivaria não somente a compreensão da importância do lazer como vivência pessoal, mas também, como futuros disseminadores dessas práticas no caso dos estudantes da área da saúde.

\section{Considerações Finais}

A partir da revisão bibliográfica é possível observar que boa parte dos estudos ainda apresenta uma visão limitada do lazer, restringindo-o em especial às práticas corporais. Por outro lado é fundamental que se amplie o espectro do que se 
compreende como lazer, tendo em vista que há trabalhos que valorizaram outros interesses do lazer associando-os positivamente à saúde dos estudantes universitários.

Ressalta-se também os achados de estudos que apontaram uma associação positiva entre a valorização do tempo livre utilizado com vivências no âmbito do lazer e melhores condições de saúde, potencializando a necessidade de criação de ambientes propícios para tais práticas, além de mais investimento em estudos nesta área.

Foi observado ainda a associação de ingestão de álcool nessa população no contexto do lazer, pontuando a necessidade de criação de estratégias para educar e incentivar o consumo mais consciente.

Por fim, diante dos achados, destaca-se a necessidade de se criar estratégias para lidar com as tensões geradas no período universitário na vida desses jovens, incentivando a adoção de uma cultura e educação voltadas para o lazer como busca de melhor qualidade de vida . É possível que estas estratégias possam influenciar suas formações profissionais fazendo com que, futuramente, estes estudantes atuem como disseminadores das vivências no contexto do lazer, para aqueles que estejam sob seus cuidados.

\section{Colaboradores}

JL Vieira, LA Romera e MCP Lima trabalharam na concepção, delineamento da pesquisa, metodologia, leitura e interpretação dos artigos, e na redação final. 


\section{Referências}

1. Torrinha F. Dicionário latino-português. Porto: Gráficos Reunidos; 1937.

2. Norbert E, Dunning E. A busca da excitação. Lisboa: Difel; 1992.

3. Martins JC, Baptista MM. O ócio nas culturas contemporâneas - Teorias e novas perspectivas em investigação. In: Martins JC, Baptista MM, organizadores. Tempo livre, ócio e lazer: sobre palavras, conceitos e experiências. Coimbra: Grácio Editor; 2013. p. 11-22.

4. Carvalho YM. Lazer e saúde: a sociedade e o social. In: Marcellino NC, organizadores. Lazer e sociedade. Campinas: Alínea; 2008. p. 105-119.

5. Dumazedier J. Valores e conteúdos culturais do lazer. São Paulo: SESC; 1980.

6. Camargo LOL. O que é lazer? São Paulo: Brasiliense; 1986.

7. Schwartz GM. O conteúdo virtual do lazer: contemporizando Dumazedier. Licere 2003, 2(6):23-31.

8. Roeder MA. Benefícios da atividade física em pessoas com transtornos mentais. Rev. Bras. Ativ. Fís. Saúde 1999, 4:62-76.

9. Araújo TM Araújo TM, Carmo Júnior JJ, Almeida MMG, Pinho PS. Prática de atividades de lazer e morbidade psíquica em residentes de áreas urbanas. Rev. Baiana Saúde Públ 2007; 31(2):294-310.

10. Vieira JLL, Porcu M, Buzzo VAS. A prática da hidroginástica como tratamento complementar para pacientes com transtorno de ansiedade. Rev. Bras. Psiquiatr 2009; 58(1):8-16.

11. Zonta R, Robles ACC, Grosseman S. Estratégias de enfrentamento do estresse desenvolvidas por estudantes de medicina da Universidade Federal de Santa Catarina. Rev. Bras. Educ. Med 2006; 30(3):147-153.

12. Rios LC, Almeida MMG, Rocha SV, Araújo TM, Pinho PS. Atividades físicas de lazer e transtornos mentais comuns em jovens de Feira de Santana, Bahia. Rev. Psiquiatr 2011; 33(2):98-102.

13. Rocha SV, Araújo TM, Almeida MMG, Virtuoso Júnior JS. Prática de atividade física no lazer e transtornos mentais comuns entre residentes de um município do Nordeste do Brasil. Rev. Bras. Epidemiol. 2012; 15(4):871-883.

14. Silva AO, Cavalcante Neto JL. Associação entre níveis de atividade física e transtorno mental comum em estudantes universitários. Motricidade 2014; 10(1):49-59.

15. Facundes VLD, Ludemir AB. Common mental disorders among health care students. Rev. Bras. Psiquiatri 2005; 27(3):194-200.

16. Lima MCP, Domingues MS, Cerqueira ATAR. Prevalência e fatores de risco para transtornos mentais comuns entre estudantes de medicina. Rev Saude Publica 2006; 40(6):1035-1041.

17. Fiorotti KP, Rossini RR, Miranda AE. Perfil dos Estudante de Medicina da Universidade Federal do Espírito Santo, 2007. Rev. Bras. Educ. Med 2010; 34(3):355-362.

18. Sousa TF. Atividades físicas praticadas no lazer por universitários de uma instituição pública do Nordeste do Brasil. Rev. Bras Ativ Fis e Saúde 2012; 17(2):125-131.
19. Mielke GI, Ramis TR, Campos E, Habeyche, Oliz MM, Germano M, Tessmer S, Azevedo MR, Hallal PC. Atividade física e fatores associados em universitários do primeiro ano da Universidade Federal de Pelotas. Rev. bras. ativ. fís. e saúde 2010; 15(1):57-64.

20. Bielemann R, Karini G, Azevedo MR, Reichert FF. Prática de atividade física no lazer entre acadêmicos de educação física e fatores associados. Rev. Bras. de atividade física \& Saúde 2007; 12(3):65-72.

21. Wolf TM, Kissling GE. Changes in Life-style Characteristics, Health, and Mood of Freshman Medical Students. J Med Educ 1984; 59(10):806-814.

22. Haase A, Steptoe A, Sallis J, Wardle J. Leisure-time physical activity in university students from 23 countries: associations with health beliefs, risk awareness, and national economic development. Prev Med 2004; 39(1):182-190.

23. Molina-Garcia J, Castillo I, Queralt A. Leisure-time physical activity and psychological well-being in university students. Psychol Rep 2011; 109(2):453-460.

24. Goldfarb AH, Hatfield BD, Armstrong D, Potts J. Plasma beta-endorphin concentration: response to intensity and duration of exercise. Med. Sci. Sports Exerc. 1990; 22(2):241-244.

25. Giovani SC, Ribeiro JL, Olivieira AR. Níveis de beta-endorfina em resposta ao exercício e no sobretreinamento. Arq. Bras. Endocrinol. Metab 2008; 52(4):589-598.

26. Nybo L, Sundstrup E, Jakobsen MD, Mohr M, Hornstrup T, Simonsen L, Bulow J, Randers MB, Nielsen JJ, Aagaard P. Krustrup P. High-Intensity Training versus Traditional Exercise Interventions for Promoting Health. Med. Sci. Sports Exerc 2010; 42(10):1951-1958.

27. Otto MW, Smits JAJ. Exercise for mood and anxiety. New York: Ed. Oxford; 2011.

28. Sousa TF, José HPM, Barbosa AR. Condutas negativas à saúde em estudantes universitários brasileiros. Cien Saude Colet 2013; 18(12):3563-3575.

29. Silva AG, Cerqueira ATAR, Lima MCP. Apoio social e transtorno mental comum entre estudantes de Medicina. Rev. Bras. Epidemiol 2014; 17(1):229-242.

30. Vieira JL, Lima MCP. Transtorno Mental Comum e lazer entre estudantes da área da saúde do campus de Botucatu -UNESP: um estudo transversal [dissertação]. Botucatu: Universidade Júlio de Mesquita; 2015.

31. Vieira EM, Barbieri CLA, Vilela DB, Lanhez Júnior E, Tomé FS, Woida FM, Martinez GL, Vicente LM, Gava NF, Lira PG, Brandão TO, Mendonça TC. O que eles fazem depois da aula? As atividades extracurriculares dos alunos de ciências médicas da FMRP-USP. Medicina 2004; 37(1/2):84-90.

32. Beuter M, Alvim NAT, Mostardeiro SCTS. O lazer na vida de acadêmicos de enfermagem no contexto do cuidado de si para o cuidado do outro. Texto Contexto Enferm 2005; 14(2):222-228.

33. Rocha GWF, Siqueira VHF. Práticas sociais de estudantes de medicina na universidade pública: celebrações, eventos e cidadania. Trab. Educ. Saúde 2009; 7(1):149165. 
34. Guareschi NMF, Wendt GW, Huning SM. Estudantes de psicologia no Brasil e o contexto sociocultural. Avaliação Psicológica 2011; 10(3):233-269.

35. Lonsdale AJ, North AC. Why do we listen to music? A uses and gratifications analysis. Br J Psychol 2011; 102(1):108-134.

36. Hodgson K, Thompson R. What do medical students read and why? A survey of medical students in Newcastle-upon-Tyne, England. Med Educ 2000; 34(8):622629.

37. Baldissera VDA, Jaques AE, Philbert LAS, Corral-Mulato S, Santos JL, Bueno SMV. As percepções de acadêmicos de enfermagem acerca do lazer. Cogitare Enferm 2011; 16(2):326-332.

38. Bradby M, Soothill K. Management of leisure time by prospective nurses. Br J Nurs 1997; 6(5):285-289.

39. Amaducci CM, Mota DDFC, Pimenta CAM. Fadiga entre estudantes de graduação em enfermagem. Rev. Esc. Enferm. USP 2010; 44(4):1052-1058.

40. Tempski P, Bellodi PL, Paro HBMS, Enns SC, Martins MA, Schraiber LB. What do medical students think about their quality of life? A qualitative study. $B M C$ Med Educ 2012; 12(106):1-8.

41. Kelly JA, Bradlyn AS, Dubbert PM, Lawrence JS. Stress Management Trainning in Medical School. J Med Educ 1982; 57(2):91-99.

42. Folse ML, DaRosa DA, Folse R. The relationship between stress and attitudes toward leisure among firstyear medical students. J Med Educ 1985; 60(8):610-617.

43. Marshall LL, Allison A, Nykamp D, Lanke S. Perceived stress and quality of life among doctor of Pharmacy students. Am J Pharm Educ 2008; 72(6):1-8.

44. Soto LFL, Torres ICS, Arévalo MTV, Cardona JAT, Sarria AR, Polanco AB. Comportamiento y salud de los jóvenes universitários: satisfacción com el estilo de vida. Pensamiento Psicológico 2009; 5(12):71-88.

45. Sanchez JRL. Programa Recreativo para la comunidade estudiantil de la Sede Universitaria Municipal - Bayamo. Revista Electrônica Granma Ciencia 2008; 12(1).

46. Carballeira M, González JA, Marrero RJ. Diferencias transculturales en bienestar subjetivo: México y España. Anales de psicologia 2015; 31(1):199-206.

47. Rosenthal TL, Montegomery LM, Shadish JWR, Lichstein KL. Leisure interest patterns and subjective stress in college students. Behav. Res. Ther. 1989; 27(1):59-64.

48. Salazar-Torres IC, Varela-Arévalo MT, Lema-Soto LFL, Tamayo-Cardona JA, Duarte-Alarcón CD. Evaluación de las conductas de salud em jóvenes universitários. Rev. Salud Pública 2010; 12(4):599-611.

49. Mesquita, AMC, Bucaretchi HA, Castel S, Andrade AG. Estudantes da Faculdade de Medicina da Universidade de São Paulo: uso de substâncias psicoativas em 1991. Revista ABP-APAL 1995; 17(2):47-54.

50. Finlay AK, Ram N, Maggs JL, Caldwell LL. Leisure activities, the social weekend, and alcohol use: Evidence from a daily study of first-year college students. J Studon alcohol Drugs 2012; 73(2):250-259.
51. Fournier AK, Ehrhart IJ, Glindemann KE, Geller ES Intervening to decrease alcohol abuse at university parties: Differential Reinforcement of intoxication level. Behav Modif 2004; 28(2):167-181.

52. Glindemann KE, Ehrhart IJ, Drake EA, Geller ES. Reducing excessive alcohol consumption at university fraternity parties: A cost-effective incentive/reward intervention. Addict Behav 2007; 32(1):39-48.

53. Baldisserotto CM, Soar Filho E, Nedel F, Sakae TM. Problemas psiquiátricos menores e indicadores do uso problemático de álcool entre os estudantes de medicina da Universidade do sul de Santa Catarina - UNISUL. Arq. Catarin. Med 2005; 34(4):73-79.

54. Lucas ACS, Parente RCP, Picanço NS, Conceição DA Costa KRC, Magalhães IRS, Siqueira JCA. Uso de psicotrópicos entre universitários da área da saúde da Universidade Federal do Amazonas, Brasil. Cad Saude Publica 2006; 22(3):663-671.

55. Tockus D, Gonçalves PS. Detecção do uso de drogas de abuso por estudantes de medicina de uma universidade privada. J. Bras. Psiquiatr 2008; 57(3):184-187.

56. Mesquita EM, Nunes AJ, Cohen C. Avaliação das atitudes dos estudantes de medicina frente ao abuso de drogas por colegas do meio acadêmico. Rev. Psiquiatry Clin 2008; 35(1):8-12.

57. Pereira DS, Souza RS, Buaiz V, Siqueira MM. Uso de substâncias psicoativas entre universitários de medicina da Universidade Federal do Espírito Santo. J. Bras. Psiquiatr. 2008; 57(3):188-195.

58. Paduani GF, Barbosa GA, Morais JCR, Pereira JCP, Almeida MF, Prado MM, Almeida NBC, Ribeiro MA. Consumo de álcool e fumo entre os estudantes da $\mathrm{Fa}$ culdade de Medicina da Universidade de Uberlândia. Rev. Bras. Educ. Med. 2008; 32(1):66-75.

59. Araújo CP, Gomes LP, Cunha MGC, Cannizza MP, Mader MS, Martins NML, Framil US, Anjos WC. Uso de álcool e psicotrópicos e o sofrimento psíquico em estudantes de medicina da Universidade Estácio de Sá. Adolesc. Saúde 2009; 6(1):28-32.

60. Petroianu A, Reis DCF, Cunha BDS, Souza DM. Prevalência do consumo de álcool, tabaco e entorpecentes por estudantes de medicina da Universidade Federal de Minas Gerais. Rev. Assoc. Med. Bras. 2010; 56(5):568571.

61. Brasil. Presidência da República. Secretaria Nacional de Políticas sobre Drogas. I Levantamento Nacional sobre o uso de álcool, tabaco e outras drogas entre universitários das 27 capitais brasileiras. Brasília: SENAD; 2010.

62. Stumbo NJ, Peterson CA. El modelo de habilidade de ócio. In: Gordeña Etxebarria S. Modelos de intervención em ócio terapêutico. Bilbao: Universidad de Deusto; 2000. p. 13-34. Documentos de Estudos de Ócio, n.11.

Artigo apresentado em 17/07/2016

Aprovado em 09/11/2016

Versão final apresentada em 11/11/2016 
